\title{
Hipotermia postquirúrgica: incidencia en el Hospital Ángeles Lomas
}

\section{Post-surgical hypothermia: incidence at Hospital Ángeles Lomas}

\author{
Ana de la Cajiga León, * Claudia P Aguirre Ibarra, * Gerardo E Álvarez Reséndiz, ${ }^{\ddagger}$ \\ Lucía Escobedo Berumen, ${ }^{\S}$ Alondra R Martínez Nápoles, * Alejandra Labra Nassar,* \\ Christian García Ezquerro, * José Rodrigo Fernández Soto*
}

\section{Resumen}

La hipotermia postquirúrgica es una complicación prevenible, con una incidencia mundial de hasta $90 \%$. Es causa de complicaciones periquirúrgicas más importantes y de una elevada morbilidad. Se investigó la incidencia de hipotermia postquirúrgica en el Hospital Ángeles Lomas, lo que dio como resultado un elevado $55 \%$ de los pacientes con temperatura por debajo de $36^{\circ} \mathrm{C}$ al llegar al Área de Recuperación Postanestésica. Se realizó la medición de la temperatura con termómetro esofágico durante la cirugía en $24 \%$ de los procedimientos, y el porcentaje de cirugías en las que se utilizó el calentamiento transquirúrgico fue de $45 \%$. La finalidad del estudio fue crear conciencia sobre la importancia de la prevención de la hipotermia y sus complicaciones, así como de la medición de la temperatura transquirúrgica como un signo vital primordial.

Palabras clave: Temperatura, hipotermia, medición de temperatura, complicaciones postquirúrgicas, anestesia, calentamiento.

\section{INTRODUCCIÓN}

En la literatura mundial se ha reportado que hasta $90 \%$ de los pacientes sometidos a cirugía presentará hipotermia en el perioperatorio. ${ }^{1,2}$ Se define como temperatura corporal central cuando se encuentra la temperatura por debajo de $36^{\circ} \mathrm{C} ; i^{1-6}$ ésta se considera una complicación completamente

\footnotetext{
* Departamento de Anestesiología, Hospital Ángeles Lomas.

₹ Jefe del Departamento de Anestesiología, Hospital Ángeles Lomas.

$\S$ Pediatría, Hospital Español.
}

Correspondencia:

Dra. Ana de la Cajiga León

Correo electrónico: anadelacajiga@hotmail.com

Aceptado: 19-06-2019.

www.medigraphic.com/actamedica

\section{Abstract}

Postoperative hypothermia is a preventable complication with a worldwide incidence of up to $90 \%$. It is cause of severe complications in the perioperative period as well as high morbidity. We investigated the incidence of postoperative hypothermia at the Hospital Ángeles Lomas, with a resulting $55 \%$ of patients presenting a temperature below $36^{\circ} \mathrm{C}$ when arriving at the post-anesthesia unit. In only $24 \%$ of surgeries, temperature is measured with a transesophagueal thermometer, and active warming during surgery is used in $45 \%$ of procedures. The main objective of the study was to stress the importance of preventing hypothermia and its complications as well as the constant measurement of temperature as a key vital sign.

Keywords: Temperature, hypothermia, temperature measurement, post-operative complications, anesthesia, warming.

prevenible del cuidado perioperatorio. Es, además, una causa de múltiples complicaciones quirúrgicas y de elevada morbilidad. Hoy en día, existen diferentes métodos de calentamiento transquirúrgico disponibles, los cuales son infrautilizados.

A partir de lo anterior, es que se realizó una revisión de la literatura, así como un trabajo de investigación para conocer la incidencia de hipotermia postquirúrgica en una institución privada y, de esta manera, se pueda crear conciencia y se mejoren las condiciones térmicas de los pacientes sometidos a cirugía.

\section{MECANISMOS DE PÉRDIDA DE CALOR EN QUIRÓFANO}

Los pacientes bajo anestesia general experimentan una serie de eventos que suelen llevar a la inhibición del control central termorregulatorio, ${ }^{7,8}$ al aumento de pérdida de calor al ambiente ${ }^{9}$ y a la ausencia de una respuesta compensa- 
toria. $^{6-8}$ La administración de fármacos anestésicos, con su consecuente vasodilatación, ${ }^{9,10}$ la exposición prolongada de la piel a soluciones y superficies frías, ${ }^{9}$ así como el ambiente quirúrgico frío por largos periodos de tiempo ${ }^{9}$ interfieren en la regulación normal de la temperatura corporal. Para mantener una adecuada homeostasis y un adecuado funcionamiento el cuerpo debe siempre estar en equilibrio entre la pérdida de calor y la producción del mismo. ${ }^{3}$

En la mayoría de los casos, cuando la temperatura desciende por debajo de $36^{\circ} \mathrm{C}$, inicia una respuesta fisiológica para prevenir la pérdida de calor a través de la vasoconstricción, sin tener que activar el mecanismo de escalofríos o shivering. ${ }^{11}$ Por un lado, éstos se activan cuando la vasoconstricción de los cortocircuitos arteriovenosos no son suficientes para mantener una adecuada temperatura central y es necesario aumentar la producción de calor. ${ }^{11,12}$ Estos cortocircuitos están mediados principalmente por la liberación de norepinefrina de las terminales nerviosas adrenérgicas. ${ }^{12}$ Por otro lado, los escalofríos postanestésicos son observados con frecuencia a la emersión de pacientes anestesiados, como un intento de restablecer o recuperar la temperatura perdida durante la cirugía, e incluso pueden elevar la tasa metabólica de dos a tres veces su valor normal. ${ }^{5,11}$

\section{MEDICIÓN DE LA TEMPERATURA}

La medición de la temperatura es obligatoria en todo paciente sometido a una cirugía que sobrepase los 30 minutos, ya sea con anestesia general o regional. ${ }^{3}$ La Norma Oficial Mexicana para la práctica de la anestesiología (NOM-006-SSA3-2011) dicta que se deberá medir la temperatura del paciente en intervalos frecuentes, a criterio del médico tratante. ${ }^{13}$

La medición central puede hacerse por vía invasiva y no invasiva. El estándar de oro para la medición central es la temperatura de la arteria pulmonar por medio de un catéter. ${ }^{14}$ Dentro de los métodos invasivos más sensibles se encuentran los valores de la membrana timpánica, que muestra la temperatura de la arteria carótida, en tanto que la temperatura de la aorta se puede medir en la nasofaringe $o$ en la parte distal del esófago. ${ }^{11,12,15}$ Los métodos no invasivos o indirectos incluyen la temperatura oral, rectal, axilar, vesical o sobre la piel. 3,10,11 El termómetro digital o los termómetros infrarrojos son una alternativa ventajosa sobre el resto, ya que brindan un resultado rápido, una mayor comodidad para el paciente, facilidad de uso y una mínima invasión. ${ }^{10,11,16}$

\section{ANESTÉSICOS E HIPOTERMIA}

La hipotermia afecta el uso de relajantes neuromusculares, como el vecuronio, el cual prolonga su duración de acción ${ }^{17}$ en pacientes con una temperatura $<34{ }^{\circ} \mathrm{C}$ y produce un retraso en el inicio de acción del fármaco por el paso de la circulación a la placa neuromuscular. ${ }^{3,17,18}$ Por otro lado, se ha visto que los halogenados utilizados en anestesia general inhiben la termogénesis, ${ }^{19}$ así como la respuesta autonómica a la hipotermia. ${ }^{20} \mathrm{El}$ propofol en infusión aumenta su concentración plasmática hasta 30\% por debajo de los $33^{\circ} \mathrm{C}$, dada por una disminución en el aclaramiento del compartimiento central hacia el periférico. ${ }^{18,21}$ Igualmente, el fentanilo aumenta su concentración plasmática $5 \%$ por cada grado descendido. ${ }^{18}$

\section{COMPLICACIONES}

Dentro de las múltiples complicaciones que surgen a consecuencia de la hipotermia, encontramos, en primer lugar, un aumento de la incidencia de infecciones del sitio quirúrgico, ya que las defensas inmunes mediadas por anticuerpos se ven afectadas. 4,8,9,22 Se da una disminución de la tasa metabólica de $5 \%$ por cada grado debajo de los $36{ }^{\circ} \mathrm{C}^{22}$ La disminución en la tensión de oxígeno a nivel subcutáneo y la misma vasoconstricción termorreguladora generan una disminución del aporte y de la disponibilidad de oxígeno en los tejidos periféricos. $4,8,9,22$

A nivel hematológico, la hipotermia causa una disfunción reversible en la disponibilidad de los activadores plaquetarios por alteraciones en la actividad del tromboxano A2, contribuyendo a cambios en su capacidad de adhesión, lo que da como resultado en una trombocitopenia por secuestro a nivel portal, ${ }^{20,22}$ aumento en la viscosidad sanguínea ${ }^{20}$ y una reducción en la función plaquetaria, dañando las enzimas de la cascada de coagulación. ${ }^{23}$ La hipotermia altera la capacidad de cicatrización de las heridas, ${ }^{4}$ y se sabe que con una reducción de $1.6^{\circ} \mathrm{C}$ puede aumentar el sangrado hasta $500 \mathrm{~mL}$ y la necesidad de una transfusión alogénica. ${ }^{4,10,22}$ Según autores como Crisóstomo y colaboradores, ${ }^{22}$ el tiempo de protrombina (TP) y el tiempo parcial de tromboplastina (TTP) se prolongan cuando la temperatura del paciente es $<33{ }^{\circ} \mathrm{C}$, ya que se altera la cascada de coagulación. ${ }^{20,22}$ Existe una desviación hacia la izquierda de la curva de disociación de la hemoglobina por cada grado disminuido, lo que causa una baja de la entrega de oxígeno a los tejidos periféricos. ${ }^{22}$ La hipotermia moderada de $<36$ ${ }^{\circ} \mathrm{C}$ aumenta significativamente la pérdida sanguínea en $16 \%$ y aumenta el riesgo de una transfusión en $22 \% .{ }^{23}$ Existe una mayor incidencia de eventos cardiovasculares y una disminución en el gasto cardiaco, ${ }^{9}$ influenciado principalmente por los escalofríos postoperatorios, los cuales aumentan el consumo de oxígeno, ${ }^{1}$ la liberación de norepinefrina ${ }^{1,9}$ y la isquemia miocárdica. 1,9,20

Otras complicaciones de la hipotermia incluyen la aparición de úlceras por presión, ${ }^{1}$ un mayor tiempo para la extubación por retraso en la recuperación anestésica, 4,6 
un mayor tiempo de recuperación, ${ }^{4,6}$ una mayor estancia intrahospitalaria, ${ }^{1,4}$ incomodidad para el paciente y una mayor mortalidad. ${ }^{9,10}$ La hipotermia aumenta la duración de la hospitalización en 20\%, sin importar si hay infección o no, ya que la recuperación per se se prolonga. ${ }^{18}$

\section{PREVENCIÓN DE LA HIPOTERMIA Y MÉTODOS DE CALENTAMIENTO}

El precalentamiento es la única técnica efectiva que ha mostrado una atenuación en la pérdida de calor por redistribución que se da posterior a la anestesia general. 3,6 La mejor forma de evitarlo es con el aire caliente forzado, el cual calienta los tejidos periféricos e induce una vasodilatación periférica, previa a la inducción, lo que aumenta la temperatura y disminuye la necesidad de paso de calor desde el compartimiento central. 3,10,24 Algunas formas frecuentes de prevención de hipotermia son los sistemas activos de calentamiento de la superficie corporal, que generan calor mecánicamente al calentar aire, agua o gel. ${ }^{8} \mathrm{El}$ precalentamiento activo debe realizarse en la sala de preanestesia de 30 a 60 minutos antes de la inducción y durante la monitorización en quirófano. ${ }^{3}$ Éste mantiene su efecto por hasta 80 minutos después de la inducción anestésica y atenúa la pérdida de calor por la redistribución posterior a la misma. ${ }^{3,6,7}$ Durante el procedimiento anestésico, la vasodilatación que se ocasiona por el aire caliente forzado en los tejidos periféricos aumenta la temperatura y disminuye la necesidad del paso de calor del compartimiento central al periférico. ${ }^{3,10,24} \mathrm{El}$ aire forzado ha demostrado disminuir el riesgo de infección del sitio quirúrgico al no interrumpir el flujo laminar del aire ni contaminar el sitio quirúrgico con el aire movilizado a nivel del piso. ${ }^{7}$
Existe también el calentamiento eléctrico/resistivo de las extremidades, torso y espalda; éste ha mostrado ser equitativo al calentamiento por aire forzado y no interfiere con los campos quirúrgicos o la preparación de la piel, además de que puede ser encendido desde antes de la transferencia del paciente a la mesa quirúrgica. ${ }^{6}$

Los líquidos intravenosos calientes comparados con líquidos a temperatura ambiente mejoran la temperatura central de los pacientes y deben calentarse a una temperatura de 38 a $40{ }^{\circ} \mathrm{C} .3,6,25$

\section{INCIDENCIA DE HIPOTERMIA POSTQUIRÚRGICA EN EL HOSPITAL ÁNGELES LOMAS}

Se realizó un estudio observacional para conocer la incidencia de pacientes que cursan con hipotermia postquirúrgica en el Hospital Ángeles Lomas, también con ello se busca saber qué método es el más utilizado para el calentamiento transoperatorio y cuál es el porcentaje de cirugías en las que se mide la temperatura transoperatoria. En la Tabla 1 se encuentran los criterios de selección utilizados para el estudio.

\section{MATERIAL Y MÉTODOS}

Previa autorización del Comité Local de Investigación y Ética del Hospital Ángeles Lomas y obteniendo consentimiento informado de cada paciente, se realizó un estudio observacional, analítico, transversal y prospectivo, para obtener la incidencia de hipotermia quirúrgica en el postoperatorio inmediato. Se midió en todos los pacientes la temperatura de la membrana timpánica utilizando un termómetro infrarrojo a su ingreso al Área de Recuperación.

Tabla 1: Criterios de selección.

Criterios de inclusión

- Pacientes ingresados para cirugía en el área de quirófano en un periodo de cuatro meses

- Pacientes con estado físico, según la American Society of Anesthesiologists (ASA), de I, II o III

- Valoración preoperatoria en la que se especifique que se utilizará anestesia general

- Pacientes que ingresen al área de recuperación postquirúrgica

- Pacientes conscientes, de cualquier género y edad

Criterios de exclusión

- Cirugía cardiaca, cirugía ginecológica, cirugía neonatal

- Pacientes con fiebre o datos de síndrome de respuesta inflamatoria sistémica
Criterios de eliminación

- Pacientes que ingresen a la Unidad Terapia Intensiva posterior al procedimiento quirúrgico

- Pacientes con fiebre postquirúrgica

- Paro cardiorrespiratorio transquirúrgico

- Pérdida de registro 


\section{RESULTADOS}

Se tomaron en cuenta 118 pacientes que cumplían criterios de inclusión en un periodo representativo de cuatro meses. De éstos, dos fueron eliminados: uno por presentar fiebre postquirúrgica y otro por ser trasladado a la Unidad de Terapia Intensiva. Los 116 pacientes restantes fueron sometidos a cirugía programada de Cirugía General, Ortopedia, Cirugía Plástica, Oncología, Otorrinolaringología, Oftalmología, Neurología y Urología en el quirófano central del Hospital Ángeles Lomas. Una vez recolectados los datos se analizaron utilizando el programa estadístico SPSS Statistics v.21.0.

Los resultados muestran que $55 \%(n=64)$ de los pacientes presenta hipotermia postquirúrgica, y del total de pacientes únicamente se precalentó a $3 \%$ en el Área de Preanestesia. Además, en 55\% $(n=64)$ de las cirugías, no se utilizó ningún método de calentamiento transquirúrgico.

El $45 \%(n=52)$ de las cirugías en las que sí se utilizó el calentamiento durante el procedimiento quirúrgico, el método más utilizado es el aire caliente forzado/cobija de aire caliente y, en segundo lugar, el colchón térmico. De estos pacientes, $28 \%(\mathrm{n}=32)$ presentó hipotermia postquirúrgica.

Se observó que únicamente se mide la temperatura transquirúrgica con termómetro esofágico en $24 \%$ ( $n=$ 28) de las cirugías realizadas; de éstas, presentó hipotermia $89 \%(n=25)$ y en $48 \%$ se utilizó calentamiento transquirúrgico $(n=13)$. Del total de cirugías en las que sí se realizó la medición de la temperatura, además de utilizar calentamiento, $36 \%(n=11)$ presentó hipotermia. Los escalofríos postquirúrgicos se presentaron en 19\% (n $=22$ ) de los pacientes.

\section{DISCUSIÓN}

Posterior a una revisión exhaustiva de la literatura, se concluyó que la hipotermia, con sus múltiples complicaciones, es un evento prevenible que se repite más de lo que debiera. Se buscó la incidencia de hipotermia postquirúrgica en el Hospital Ángeles Lomas para evidenciar la frecuencia con la que ocurre este evento, para posteriormente lograr un cambio en su prevención. El 55\% de los pacientes sometidos a cirugía presentaron hipotermia, lo que va a acorde con las cifras reportadas en la literatura mundial; este número podría variar en caso de expandir el estudio incluyendo a más pacientes y a otras especialidades quirúrgicas, como Ginecología u otras áreas, donde se administra anestesia fuera de quirófano, tales como hemodinamia o resonancia magnética.

La única manera efectiva de prevenir la hipotermia postquirúrgica es el precalentamiento del paciente, ${ }^{3,6}$ aun- que solamente $3 \%$ de la población se precalentó durante la realización del estudio, lo que se traduce en la falta de información acerca de la importancia del precalentamiento y las complicaciones que podrían evitarse con la utilización de aire forzado caliente, previo al traslado de los pacientes al quirófano.

Se cuenta con múltiples recursos para el calentamiento pre- y transquirúrgico de los pacientes, entre ellos, el aire caliente forzado, el calentamiento eléctrico/resistivo (incluidas las mantas eléctricas, colchones eléctricos, líquidos intravenosos calientes) y el calentamiento de la sala quirúrgica. No obstante, se ha observado que en $55 \%$ de las cirugías realizadas no se utiliza ningún método. Se debe crear conciencia y hacer hincapié sobre la importancia de utilizar el método de calentamiento ideal para cada paciente.

Se observó que, a pesar de contar con el calentamiento transquirúrgico, $28 \%$ de estos pacientes presentó temperaturas por debajo de $36{ }^{\circ} \mathrm{C}$ al salir al área de Cuidados Postanestésicos. Rara vez se mide la temperatura a los pacientes antes o después de la cirugía como protocolo estandarizado en el que se midan los signos vitales; cambiar esto podría ayudar a prevenir la hipotermia y sus consecuentes complicaciones. Únicamente 19\% de los pacientes presentó shivering o escalofríos postoperatorios, los cuales, por sí mismos, pueden aumentar el riesgo del paciente posterior a la cirugía. Si los médicos o personal de enfermería encargados de los pacientes postoperados no miden la temperatura de forma rigurosa y únicamente se guían por la presencia o no de shivering para utilizar alguna forma de calentamiento activo, la mayor parte de los pacientes podría presentar hipotermia inadvertida.

A pesar de que están disponibles termómetros esofágicos en las salas de quirófano, únicamente se utilizaron en $24 \%$ de los procedimientos. Teniendo los recursos, es de suma importancia que los médicos hagan el hábito de registrar la temperatura durante el procedimiento quirúrgico como parte de la medición habitual de los signos vitales. En $48 \%$ de las cirugías en las que se registró la temperatura, sí se utilizó calentamiento transquirúrgico, lo que puede traducirse a que los anestesiólogos que miden la temperatura durante la cirugía están al pendiente de que sus pacientes mantengan una adecuada temperatura corporal.

Aunque en la gran mayoría de las cirugías en las que se midió la temperatura se utilizó calentamiento transquirúrgico, un impresionante 36\% de éstas presentó hipotermia al salir de quirófano, lo que hace pensar que el método de calentamiento no está siendo utilizado debidamente, por el tiempo suficiente o porque debe ser utilizado en conjunto con otro. 
La recomendación para mejorar la calidad de este estudio sería ampliar la muestra de pacientes para así poder lograr resultados con mayor significancia estadística, y correlacionarlo con el tiempo quirúrgico y los signos vitales; igualmente, medir la sedación postquirúrgica y compararla con la temperatura, así como preguntar directamente si los pacientes refieren frío. Finalmente, también es importante realizar el seguimiento y el tratamiento de las complicaciones en los pacientes que presenten hipotermia ahora que se conoce la incidencia de este fenómeno en nuestra institución.

\section{CONCLUSIÓN}

La hipotermia postquirúrgica es una complicación del cuidado perioperatorio completamente prevenible, la cual puede causar múltiples complicaciones en el periodo postoperatorio. La incidencia de hipotermia postquirúrgica en el Hospital Ángeles Lomas es de 55\% y el método de calentamiento más utilizado es el aire caliente forzado, el cual se ha posicionado como el mejor método para prevenir la hipotermia durante el periodo prequirúrgico, pero está infrautilizado.

Se debe realizar la medición de la temperatura, el precalentamiento para la prevención y el adecuado control de su temperatura durante el transquirúrgico en todo paciente sometido a cirugía.

\section{REFERENCIAS}

1. Boet S, Patey A, Baron J, Mohamed K, Pigford A, Bryson G et al. Factors that influence effective perioperative temperature management by anesthesiologists: a qualitative study using the Theoretical Domains Framework. Can J Anesth. 2017; 64 (6): 581-596.

2. Mehta $\mathrm{OH}$, Barclay KL. Perioperative hypothermia in patients undergoing major colorectal surgery. ANZ J Surg. 2013; 84 (7-8): 550-555.

3. Fernández-Meré LA, Álvarez-Blanco M. Manejo de la hipotermia perioperatoria. Revista Española de Anestesiología y Reanimación. 2012; 59 (7): 379-389.

4. Gurunathan U, Stonell C, Fulbrook P. Perioperative hypothermia during hip fracture surgery: an observational study. J Eval Clin Pract. 2017; 23 (4): 762-766.

5. Young $\mathrm{VL}$, Watson ME. Prevention of perioperative hypothermia in plastic surgery. Aesthetic Surg J. 2006; 26 (5): 551-571.

6. John M, Ford J, Harper M. Peri-operative warming devices: performance and clinical application. Anaesthesia. 2014; 69 (6): 623-638.
7. Warttig S, Alderson P, Campbell G, Smith A. Interventions for treating inadvertent postoperative hypothermia. Cochrane Database Syst Rev. 2014; (11): CD009892.

8. Madrid E, Urrútia G, Roqué I, Figuls M, Pardo-Hernandez H, Campos J, Paniagua P et al. Active body surface warming systems for preventing complications caused by inadvertent perioperative hypothermia in adults. Cochrane Database Syst Rev. 2016; 4: CD009016.

9. Roberson MC, Dieckmann LS, Rodriguez RE, Austin PN. A review of the evidence for active preoperative warming of adults undergoing general anesthesia. AANA JL. 2013; 81 (5): 351-355.

10. Hopf H. Perioperative temperature management. Anesthesiology. 2015; 122 (2): 229-230.

11. Feinstein L, Miskiewicz M. Perioperative hypothermia: review for the anesthesia provider. The Internet Journal of Anesthesiology. 2009; 27 (2) [On line].

12. Sessler D. Temperature monitoring: the consequences and prevention of mild perioperative hypothermia. Southern African Journal of Anaesthesia and Analgesia. 2014; 20 (1): 25-31.

13. SDESEN Coahuila. Norma Oficial Mexicana NOM-006-SSA3-2011 para la práctica de la anestesiología. Diario Oifical de la Federación. México; 2012.

14. Fulbrook P. Core body temperature measurement: a comparison of axilla, tympanic membrane and pulmonary artery blood temperature. Intensive Crit Care Nurs. 1997; 13 (5): 266-272.

15. Frank S. Consequences of hypothermia. Current Anaesthesia \& Critical Care. 2001; 12 (2): 79-86.

16. Gerensea $\mathrm{H}$, Murugan R. Is there significant difference between digital and glass mercury thermometer? Advances in Nursing. 2016; 2016: $1-10$.

17. Heier T, Caldwell J. Impact of hypothermia on the response to neuromuscular blocking drugs. Anesthesiology. 2006; 104:10701080.

18. Doufas AG. Consequences of inadvertent perioperative hypothermia. Best Pract Res Clin Anaesthesiol. 2003; 17 (4): 535-549.

19. Zeba S, Surbatovic M, Marjanovic M, Jevdjic J, Hajdukovic Z, Karkalic $R$ et al. Efficacy of external warming in attenuation of hypothermia in surgical patients. Vojnosanit Pregl. 2016; 73 (6): 566-571.

20. Bayter-Marín J, Rubio J, Valedón A, Macías Á. Hypothermia in elective surgery: The hidden enemy. Rev Colomb Anesthesiol. 2017; 45 (1): 48-53.

21. Leslie K, Sessler D, Bjorksten A, Moayeri A. Mild hypothermia alters propofol pharmacokinetics and increases the duration of action of atracurium. Anesth Analg. 1995; 80 (5): 1007-1014.

22. Crisóstomo-Pineda, M, Hernández-Pérez A, Ordóñez-Espinosa G, Riera-Kinkel C. La hipotermia y sus efectos durante la anestesia en niños. Rev Mex Pediatr. 2011; 78 (4): 131-138.

23. Rajagopalan S, Mascha E, Na J, Sessler D. The effects of mild perioperative hypothermia on blood loss and transfusion requirement. Anesthesiology. 2008; 108 (1): 71-77.

24. Torossian A, Bräuer A, Höcker J, Bein B, Wulf H, Horn E. Preventing inadvertent perioperative hypothermia. Dtsch Arztebl Int. 2015; 112 (10): 166-172.

25. Intervenciones Preventivas Para la Seguridad en el Paciente Quirúrgico. México: Secretaría de Salud; 2013. 